\title{
Calibration and validation of the Mini-fiber EC image analysis instrument mean fiber diameter through direct or primary measurements
}

\begin{abstract}
The Minifiber EC (MFEC) is a portable instrument for measuring the diameter of animal fibers. Its accuracy and precision have been estimated but by comparing its measurements with those of laboratory devices that had been calibrated on other devices in turn, not on a direct or primary measure of diameter. This work attempts to test direct measurements by gravimetry, Vernier mini caliper, microscope and the classic microprojector, using a nondeformable, high resistance synthetic fiber (Kevlar) for direct measurement. The MFEC instrument is calibrated with each mean fiber diameter obtained in direct measurements and its results are compared. The conclusions drawn are that it is possible to calibrate the MFEC instrument with direct measurements on Kevlar and measurement accuracy or tolerance of 0.28 microns is obtained. This indicates a very low biased mean fiber diameter measurement by MFEC.
\end{abstract}

Volume 7 Issue 6 - 2021

\author{
Eduardo Frank, ${ }^{1,2}$ EN Hick, ${ }^{1,2}$ MVH Castillo, ${ }^{1,2}$ \\ Gaut MC, ${ }^{3}$ RH Mamani-Cato ${ }^{4}$ \\ 'IRNASUS-UCC-CONICET, Argentina \\ ${ }^{2}$ Sede UCHA-UNLAR, Argentina \\ ${ }^{3}$ Facultad de Ciencias Agropecuarias, Universidad Católica de \\ Córdoba (UCC), Argentina \\ ${ }^{4}$ INIA, Dir. Desarrollo Tecnológico Agrario, Est. Exp.Agraria \\ ILLPA, Puno, Perú
}

\begin{abstract}
Correspondence: Eduardo Frank, PhD., IRNASUS-UCCCONICET,Armada Argentina 3555, Córdoba, Argentina, Email frank.agro@ucc.edu.ar
\end{abstract}

Received: November 28, 2021 | Published: December 13, 2021 
show the same results. In the fields of science and engineering, the accuracy of a measurement system is the degree of closeness of measurements of a quantity to that quantity's true value..$^{23}$ The precision of a measurement system, related to reproducibility and repeatability, is the degree to which repeated measurements under unchanged conditions show the same results. ${ }^{23,24}$ Although the two words precision and accuracy can be synonymous in colloquial use, they are deliberately contrasted in the context of the scientific method.

The field of statistics, where the interpretation of measurements plays a central role, prefers to use the terms bias and variability instead of accuracy and precision: bias is the amount of inaccuracy and variability is the amount of imprecision.

A measurement system can be accurate but not precise, precise but not accurate, neither, or both. For example, if an experiment contains a systematic error, then increasing the sample size generally increases precision but does not improve accuracy. The result would be a consistent yet inaccurate string of results from the flawed experiment. Eliminating the systematic error improves accuracy but does not change precision.

A measurement system is considered valid if it is both accurate and precise. Related terms include bias (non-random or directed effects caused by a factor or factors unrelated to the independent variable) and error (random variability).

The terminology is also applied to indirect measurements - that is, values obtained by a computational procedure from observed data.

In addition to accuracy and precision, measurements may also have a measurement resolution, which is the smallest change in the underlying physical quantity that produces a response in the measurement.

In numerical analysis, accuracy is also the nearness of a calculation to the true value; while precision is the resolution of the representation, typically defined by the number of decimal or binary digits.

In the course of discussing, the precision of the mean when measuring fiber diameter by the projection- microscope, pay attention to an implicit assumption, found in standards covering this method, that there are no errors of observation and no errors of sampling. ${ }^{25}$ This, of course, is a commonly encountered assumption, for few statistics texts satisfactorily partition errors in measurement from variations in the variate itself.

In general, metrological analysts are concerned, more than the exactitude itself, the bias, that makes both precision and accuracy, but they are hardly differentiable. ${ }^{23}$ On the other side, bias may have little or no effect on precisión when a large number of measurements have been made. But it has a higher effect upon acuracy because it is the result of the systematic or determinate error, while the precision is the product of the random or indeterminate error, that is because it is reduced with an increase in $n$. Bias can exist between measurements by secondary systems, but unless can be confirmed by reference to a primary measuremente systems. Bias can be the results of several cuases. ${ }^{15}$ In this cases only instrumental and method errors are of interest for the validation of MF EC.

One of the principal objectives of standardiseng wool testing systems is the elimination or at least the minimizetion of bias. The use of special fiber standards to calibrate devices is not common; the calibration standards are wool samples (interlabwool).
In direct measurement methods there are some problems to measure natural fibers, for example, low resistance to compression, in the case of measurement with microcaliber, the high degree of elasticity to correctly measure the length of the individual fiber, and the variation of specific gravity, in the case of the gravimetric method. ${ }^{5}$ For this reason, synthetics were selected to perform the determination of direct measurements to generate standards for the MiniFiber EC. An aramid fiber (aromatic and polyamide) commercially called kevlar was chosen.

The reasons for choosing this fiber were several:

a. Textile behavior similar to animal fibers: Foldable and with artificial crimp, almost circular section, similar to animal fibers, narrow diameter range: $4-16 \mu \mathrm{m}$ similar to very fine animal fibers (vicuña, cashmere, ultrafine wool)

b. Very low diameter (around $12 \mu \mathrm{m}$ ) with low coefficient of variation $(\approx 12 \%)$

c. High Young's modulus ( $\approx 480$ Gpas versus 4-6 in animal fibers)

d. High tensile strength and shear force derived from the above property

e. Diameters supplied by manufacturers according to brand:

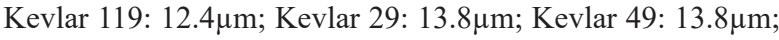
Kevlar 149: $11.5 \mu \mathrm{m}$ and Technora: $12.8 \mu \mathrm{m}$.

\section{f. Source of published information: Kawabata ${ }^{5}$ in J. Text. Inst. ${ }^{18}$}

The objective is to calibrate the MF EC instrument on a direct measurement of a non-deformable synthetic fiber that allows direct measurements of its thickness, and validate by comparisson between others instrument measure of different type of animal fibres.

\section{Materials \& methods}

\section{Direct measurement processes}

MiniFiber EC (MFEC) estimates measurements from algorithms and from measurements in pixel image analysis, therefore, they are indirect or secondary measurement systems and their measurement is subject to both accuracy and precision, but only the latter can be obtained of the direct determination of the apparatus. Only the reproducibility, taking the measurement of another device that does not measure directly, approximates this determination. Only the comparison with direct measurement systems can provide an estimate of the accuracy of each measurement, in each device and according to the types of fiber they measure.

Direct measurements are those made with the mini-caliper and the gravimetric determination of the diameter. The use of the microprojector or lanameter can also be considered a direct measure as well as the measurement with a microscope equipped with a millimeter ruler that can be calibrated to microns through a template. The one used is the Neubauer-Thoma hematology chamber. Although in any case it is not an estimate through an established function but through a fixed conversion factor from micrometric units to microns $(\mu \mathrm{m})$.

The minicalibre or minimicrometer (Minical): In international units (IS) the primary unit of measurement of length is the meter. A number of devices such as the micrometer and the micrometer caliper (Vernier) are used to measure widths or thicknesses, in fractions of a 
meter, in various materials. It is possible to measure distances between within appropriate materials on the order of 1.0 micrometers. The thickness is determined by using the high precision arrangement of the micrometer screw to adjust distances between the parallel jaws of the device that captures the material transversely. The screw provides a method to amplify the scale and perform the fine adjustment necessary to adjust the distances that remain between both parts of the jaw by squeezing the material. ${ }^{5}$ The use of this device makes it possible to measure the variation in diameter along the fiber, but expecting a small crushing action on the fiber, which is why it tends to measure finer than microscopic methods. ${ }^{26}$ The mini gauge used in this work defines (sensitivity) down to one micron $(1 \mu \mathrm{m})$. The mini Vernier was mounted on a clamping device provided with 2 plastic jaws that rests on the table, in this way and holding another operator, the isolated and verified fiber under a stereoscopic magnifying glass (30x) is measured at the moment that the fiber captured by the jaws no longer move. The reference of the 3 'clicks' recommended by the manufacturer is not used because that collapses or contracts the fiber despite the shear strength of the aramid fiber. ${ }^{18}$

The gravimetric method had been suggested to define its units of diameter as the result of the weight (milligrams) of 10 meters in length of an indeterminate number of fibers at a regain of $18.5 \%$ and when comparing it with microscopic measurements. Very low discrepancies are verified at fine diameter and the difference increases as the diameter increases in wool, probably due to the distance from circularity on the part of the thicker fibers. ${ }^{27}$ Later applications of this method use the relationship between mass, volume and density to define the fineness of fibers in terms of their cross section, which can be assimilated to the concept of linear density that is commonly used for tapes and threads in the textile industry.

According to this definition, the following complementary formulas can be established:

$$
\text { Density }=\frac{\text { Mass }}{\text { Volume }}=\frac{\text { Mass }}{\text { Lenth } \times \text { Area }} \gg \text { Area }=\frac{\text { Mass }}{\text { Density } \times \text { Lenth }}
$$

To calculate the final diameter of the fibers measured in length and heavy, assuming circularity and uniform density throughout and between fibers, the following equations were used:

$$
D_{g}^{2}=\frac{4}{\pi} * \frac{m}{\rho * l}
$$

Where from

$\mathrm{D}^{2} \mathrm{~g}$ : mean diameter equivalent to a circle

m: mass of the sample fibers

1: total length of the sample fibers

$\mathrm{p}$ : specific density of the fiber: kevlar $=1.44 \mathrm{~cm}^{3} / \mathrm{g}$.

Hence the mean fiber diameter of the sample or the fineness of the sample can be finally calculated as:

$$
D_{g}=\sqrt{\frac{4}{\pi} * \frac{m}{\rho * l}}
$$

The total length of the fibers was determined with a common Vernier caliper that defines up to $100 \mu \mathrm{m}$, as a decimal of the millimeter, and was weighed with a Metler-Toledo precision balance with an accuracy of $0.0001 \mathrm{~g}$.
Observing under a stereoscopic magnifying glass (30x) the washed and conditioned sample of aramid fibers, 5 different fibers were identified based on the separation that the eye makes at this magnification. Clearly observing that the fibers do not overlap or overlap, they were separated (dissected) on a velvet cloth to measure with the minicraft, and then cut into 2-millimeter pieces and mounted on coverslip slides fixed with synthetic Canada balsam.

For the gravimetric determination the fibers were separated under the magnifying glass but without discriminating by appreciable thickness, and they were measured one by one with the caliper, using the device that measures depth, since it allows to separate the jaws but from the external lake. Its allows to accurately locate the tips of the fibers and being metallic, the gauge allows pressing and keeping the fiber stretched, smoothing the artificial waves that Kevlar has. After measuring the length of 150 fibers with an average of $3.52 \mathrm{~cm}$, they were weighed on the precision mass balance, supported on a filter paper for which the balance had been arrested.

In the measurements of the respective instruments, a number of measurements was established that were sufficient to achieve an error of $5 \%(\mathrm{n} 5 \%)$, according to the following function:

$$
n \geq\left(\frac{2 \times Z_{(1-\alpha / 2)} \times \sigma}{c}\right)^{2}
$$

Were:

$\mathrm{n} \geq$ : Sample size so that the amplitude of IC does not exceed ' $\mathrm{c}$ units, that is: $\mathrm{UL}-\mathrm{LL} \leq \mathrm{c}$

\section{Z: z score (95\%): 1.96 \\ $\sigma:$ standard deviations of simple}

c: is $n 5 \%$ : IC amplitude that does not exceed ' $c$ ' units, that is: UL LL c: It can be a function of the mean: $\bar{X} x f$

The standard error of the sample mean is calculated as the standard deviation divided by the square root of the sample size.

Relative standard error $(\mathrm{SE} / \mu \mathrm{m})$ : it is the standard error divided by the sample mean, it means the implication of the mean discrepancy of the estimated mean with respect to the population mean. Ex: $\mathrm{EE} / \mu \mathrm{m}=0.2$ means that the calculated sample mean has an average discrepancy of $20 \%$ with respect to the estimated population mean.

\section{Results}

\section{Data from direct measurements}

In Table 1 (Data from direct measurements) the mean diameters obtained when calculated with direct methods were presented. The measurements obtained by calculating with a mini caliper, gravimetric method and the microscope equipped with a micrometric rack are significantly lower than those obtained with a microprojector or lanameter or by estimating weighted variables arising from the 5 types of fibers chosen to measure with the mini caliper. It is evident that the weighting method provides a similar mean but by considerably reducing the variance it achieves significant differences, this is reflected in the low confidence interval that it provides $(0.0631)$ versus the CIs of the other methods. 
Table I Average Kevlar fiber diameters measured directly with the following devices: Gravimetric (Grav), Microprojector (Microp), Microscope (Mic), Mini Vernier Caliper (MiniCal), MiniCal Weighted Average (MiniCalW)

\begin{tabular}{|c|c|c|c|c|c|c|c|c|c|c|}
\hline Source variable & $\mathbf{n}$ & Average $(\mu \mathrm{m})$ & S.D. & Min & $\operatorname{Max}$ & $P(05)$ & $P(95)$ & $n: 5 \%$ & Tr\% & $\mathrm{SE} / \mu \mathrm{m}$ \\
\hline Minical & 140 & 11.97 & 1.3 & 8.7 & 14.92 & 9.68 & 14.15 & 72 & 3.73 & 0.92 \\
\hline Microp & $13 \mid$ & 12.29 & 2.58 & 5.96 & 17.92 & 7.93 & 16.6 & 271 & 7.05 & 1.83 \\
\hline Mic & 140 & 12.32 & 1.7 & 7.54 & 16.94 & 9.59 & 15.14 & 117 & 4.51 & 1.17 \\
\hline Total & 685 & 12.15 & 0.23 & & & & & & & \\
\hline
\end{tabular}

\section{Reference:}

$\mathrm{N}$ : number of fibers measured for each device

$\mathrm{n}: 5 \%$ : necessary number of fibers measured to achieve an error equal to or less than $5 \%$

Table I: Kevlar fiber mean diameters obtained from the direct measurement of different devices: gravimetric (rav), microprojector (Microp), microscope (Mic), mini Vernier caliper (MiniCal) and MiniCal weighted average (MiniCalW)

Tr\%: percentage error actually obtained (true) with the actual measurements performed

$\mathrm{SE} / \mu \mathrm{m}$ : standard error of the mean over the mean in microns

Grav and Microp should have had more measurements, because the measurements made did not reach a percentage error of $5 \%$. In short, the difference was not very noticeable; because it still reached $7 \%$ approx. Measurements from the other devices (Mic, MiniCal, and MiniCalW) far exceeded the minimum amount because the resulting error was well below 5\%. Even so, the $\mathrm{SE} / \mu \mathrm{m}$ ratio shows that in some devices, $\sim 2 \%$ of the cases can be expected to deviate from the mean with respect to the population estimate and in the others, the possible error is even much lower.
In Figure 1 the distribution of the diameters of the fibers measured by the different devices are graphed. The 10-12 $\mu \mathrm{m}$ diameter interval is the highest frequencies in all devices, but some differences can be seen nonetheless. In all of them the interval $10-12 \mu \mathrm{m}$ falls in the following frequencies: MinCal: 0.58 Microp: 0.67 MinCalW: 0.64 Grav: 0.52 , Mic: 0.55 . The values provided are approximate because they were downloaded from the frequency bar graph that the MFEC displays on the screen.

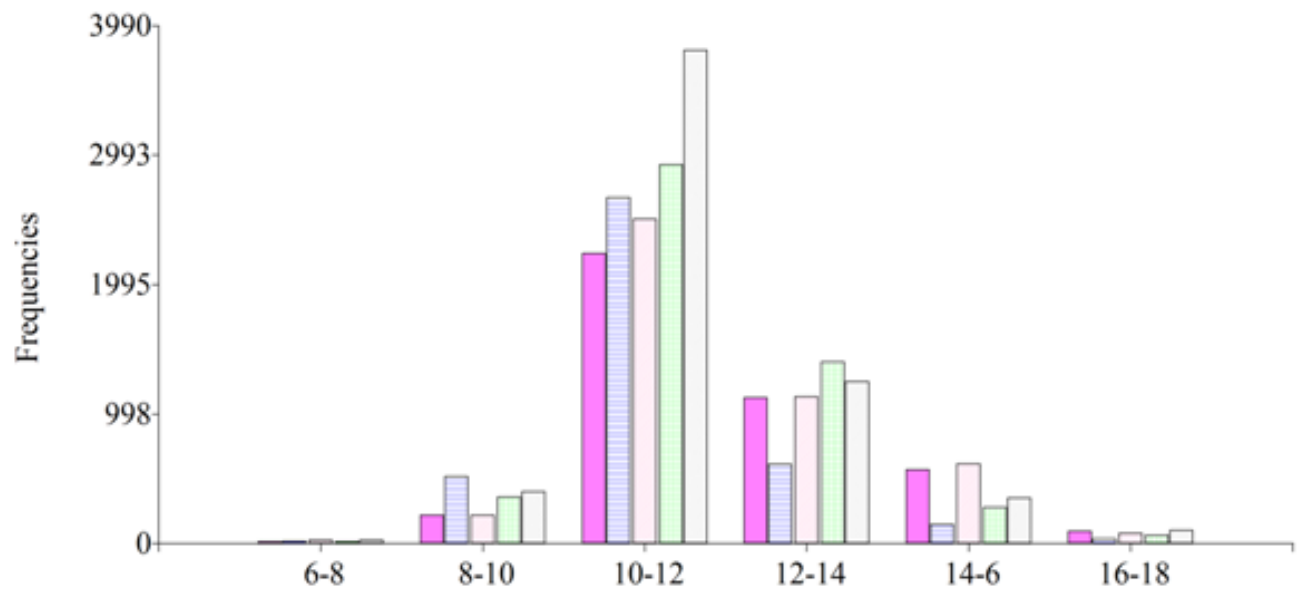

Fiber Diameter intervals

Microp

\section{Mic}

MiniCal

Figure I A graphical distribution of the frequencies of the fiber diameter intervals.

From the observations of Mic and Microp it follows that there are no fibers finer than $6 \mu \mathrm{m}$ or thicker than $18 \mu \mathrm{m}$ in this Kevlar sample. In Figure 1 there are no other fiber diameters, neither outside nor inside, therefore all the devices correctly measured the diameters without causing dummies by overlapping (thicker) or by partial measurement (finer) or even reduced by crushing, in the case of MiniCal.
The Table 2 shows an inference based on a sample using the T-test for a mean. It allows testing hypotheses about the value of the expectation of a random variable, in this case the general mean of all device measurements. Mean value under the null hypothesis: $12.15 \mu \mathrm{m}$, is the overall mean fiber diameter evidenced in Table 1. 
Table 2 Comparison of mean diameters measured in the different devices against the general mean diameter

\begin{tabular}{llllllll}
\hline Variable & $\mathbf{n}$ & Average $(\boldsymbol{\mu m})$ & SD & DL(95) & UL(95) & T & p(Bilateral) \\
\hline Grav & 134 & 11.85 & 2.59 & 11.41 & 12.29 & -1.34 & 0.1835 \\
Minical & 140 & 11.97 & 1.3 & 11.75 & 12.18 & -1.68 & 0.0951 \\
Microp & 131 & 12.29 & 2.58 & 11.84 & 12.73 & 0.62 & 0.5386 \\
Mic & 140 & 12.32 & 1.7 & 12.03 & 12.6 & 1.17 & 0.2423 \\
MinicalW & 140 & 12.33 & 0.24 & 12.29 & 12.37 & 8.95 & $<0.0001$ \\
\hline
\end{tabular}

Except for MiniCalW, none of the other measurements shows a significant difference $(\mathrm{p}<0.05)$ with the mean used as the null hypothesis in the comparison.

Another possible comparison can be made between the measurement of one device and the average of the others. Taking the data from one device and combining the others, a T-test for independent samples can be made. This allows verifying the behavior of each device in relation to the others as a whole. This is shown in Table 3.

Table 3 Comparison between one device measure (I) and the average of anothers (2) by T Student

\begin{tabular}{|c|c|c|c|c|c|c|c|c|}
\hline \multirow{2}{*}{$\begin{array}{l}\text { One device (I) } \\
\text { Grav }\end{array}$} & \multirow{2}{*}{$\begin{array}{l}\text { Another devices together (2) } \\
\text { Mic, Microp, Miinical, MiniCalW }\end{array}$} & \multicolumn{2}{|c|}{ Aver. I Aver. 2} & \multirow{2}{*}{$\begin{array}{l}\text { Diff } \\
-0.71\end{array}$} & \multirow{2}{*}{$\begin{array}{l}\text { pHomVar } \\
<0.0001\end{array}$} & \multirow{2}{*}{$\begin{array}{l}\mathbf{T} \\
-2.66\end{array}$} & \multirow{2}{*}{$\begin{array}{l}\text { fd } \\
167\end{array}$} & \multirow{2}{*}{$\begin{array}{l}\text { p-value } \\
0.0086\end{array}$} \\
\hline & & 11.53 & 12.24 & & & & & \\
\hline Microp & Grav, Mic, Miinical, MiniCalW & 11.98 & 12.12 & -0.14 & $<0.0001$ & -0.52 & 167 & 0.6022 \\
\hline Miinical & Grav, Mic, Microp, MiinicalW & 12.08 & 12.1 & -0.03 & $<0.0001$ & -0.19 & 456 & 0.8514 \\
\hline MiinicalW & Grav, Mic, Microp, Miinical & 12.36 & 12.03 & 0.33 & $<0.0001$ & 3.19 & 598 & 0.0015 \\
\hline Mic & Grav, Microp, Miinical, MiniCalW & 12.53 & 11.99 & 0.54 & 0.0055 & 3 & 253 & 0.003 \\
\hline
\end{tabular}

pHomVar: p-value variance homogeneity

$\mathrm{fd}$ : freedom degree reduction due to non homogeneity of variance

The homogeneity of variance significant is due to the difference in degrees of freedom between observation (1) and (2). However, the degrees of freedom that result from the corresponding reduction are sufficient to make valid the p-value of the Student's test for comparison of means. The average of the total differences between the measurements of the different devices is around $0.43 \mu \mathrm{m}$, but when the results of the significant comparisons are averaged alone, it reaches $0.72 \mu \mathrm{m}$, while only the non-significant ones (Microp and MiniCal) the difference between the comparisons it is only $0.10 \mu \mathrm{m}$, or practically zero.

These tests (Table 2) (Table 3) were developed because the Student's t-pair comparison was not possible. The output of the individual measurements of the fibers is not available in MFEC and the probability that in successive measurements of the same sample the same sites of the same fibers are measured is not known.

\section{Comparison between measurements of Mini Fiber, calibrated over direct measurements}

Table 4 (Comparison between direct measurements of Mini Fiber, calibrated over direct measurements) shows the comparison between direct measurements in pixel of the Mini Fiber and the different calibrations performed by direct measurements. The objective of this test was to verify if the calibration could affect the direct measurement in pixel and how it is verified in the Table 4, no significant differences $(p<0.05)$ are obtained. Only the differences in absolute values that indicate that the device when scanning measures points at random, hence the absolute difference but the non-significance $(\mathrm{p}>0.05)$ indicates the effect of chance.

Table 4 Comparison between MFEC measurements, calibrated on the direct measurements of Grav, Microp, MiniCal and MiniCalpW

\begin{tabular}{lllllllll}
\hline Variable & Group(I) & Group(2) & Aver(I) & Aver(2) & Diff & pHomVar & T & p-value \\
\hline Pixel & Grav & Microp & 8.25 & 8.23 & 0.02 & 0.1546 & 0.97 & 0.3716 \\
Pixel & Grav & MiniCalW & 8.25 & 8.26 & -0.01 & 0.2973 & -0.45 & 0.6658 \\
Pixel & Grav & MiniCal & 8.25 & 8.24 & 0.01 & 0.3142 & 0.62 & 0.5598 \\
Pixel & Microp & MiniCal & 8.23 & 8.26 & -0.03 & 0.6666 & -1.13 & 0.3006 \\
Pixel & Microp & MiniCalW & 8.23 & 8.24 & -0.01 & 0.6387 & -0.42 & 0.6922 \\
Pixel & MiniCal & MiniCalW & 8.26 & 8.24 & 0.02 & 0.9686 & 0.85 & 0.4274 \\
$\mu \mathrm{m} / \mathrm{pxl}$ & Grav & Microp & 1.46 & 1.42 & 0.04 & 0.84 & 2.74 & 0.0336 \\
$\mu \mathrm{m} / \mathrm{pxl}$ & Grav & MiniCalW & 1.46 & 1.43 & 0.03 & 0.5194 & 1.39 & 0.2126 \\
$\mu \mathrm{m} / \mathrm{pxl}$ & Grav & MiniCal & 1.46 & 1.42 & 0.04 & 0.101 & 3.35 & 0.0153 \\
\hline
\end{tabular}




\begin{tabular}{|c|c|c|c|c|c|c|c|c|}
\hline Variable & Group(I) & Group(2) & Aver(I) & Aver(2) & Diff & pHomVar & $\mathbf{T}$ & p-value \\
\hline$\mu \mathrm{m} / \mathrm{pxl}$ & Microp & MiniCal & 1.42 & 1.43 & -0.01 & 0.4026 & -0.65 & 0.5387 \\
\hline$\mu \mathrm{m} / \mathrm{pxl}$ & Microp & MiniCalW & 1.42 & 1.42 & 0 & 0.1405 & -0.13 & 0.8992 \\
\hline$\mu \mathrm{m} / \mathrm{pxl}$ & MiniCal & MiniCalW & $\mathrm{I} .43$ & 1.42 & 0.01 & 0.0328 & 0.66 & 0.558 \\
\hline
\end{tabular}

pHomVar: p-value variance homogeneity

$\mathrm{fd}$ : freedom degree reduction due to non homogeneity of variance was not indicated due to absence of significant difference

\section{Data of direct and indirect measurements and the comparison between them}

In Table 5 (Data of direct and indirect measurements and the comparison between them), the direct measurements (DMF) with the measurements of Mini Fiber EC calibrated by the parameters of each direct measurement (Minical, weighted Minical, Microprojector, Microscope and gravimetric method) (MDF $(\mu \mathrm{m})$. Only very significant difference $(\mathrm{p}<0.001)$ is observed in the calibration of weighted Mini caliber and significant with microprojector $(p<0.05)$. The average difference between the Calibrated measurements of the Mini Fiber and the direct measurements (were significantly different or not) resulted in $0.32 \mu \mathrm{m}$, only non signficiant is $0.28 \mu \mathrm{m}$ and only signficative different is $0.38 \mu \mathrm{m}$, being able to establish that this magnitude would be what we call device accuracy.

Table 5 Comparison by independent T Student test of fiber diameter average (MFD) between diferent device calibrations on MFEE

\begin{tabular}{lllllllll}
\hline Variable & Group I & Group 2 & Aver(I) & Aver(2) & Diff & pHomVar & T & p-valor \\
\hline DMF & Mic & MiniCal & 12.73 & 11.97 & 0.76 & 0.0238 & 3.83 & 0.0002 \\
DMF & MiniCal & MiniCalW & 11.97 & 12.35 & -0.39 & $<0.0001$ & -3.02 & 0.003 \\
DMF & Mic & MiniCalW & 12.73 & 12.35 & 0.38 & $<0.0001$ & 2.42 & $0.017 \mid$ \\
DMF & Grav & Mic & 12.1 & 12.73 & -0.63 & $<0.0001$ & -2.17 & 0.0314 \\
DMF & Mic & Microp & 12.73 & 12.2 & 0.53 & $<0.0001$ & 1.69 & 0.0926 \\
DMF & Microp & MiniCal & 12.2 & 11.97 & 0.23 & $<0.0001$ & 0.76 & 0.4496 \\
DMF & Grav & MiniCal & 12.1 & 11.97 & 0.13 & $<0.0001$ & 0.46 & 0.6447 \\
DMF & Grav & Microp & 12.1 & 12.2 & -0.1 & 0.2653 & -0.27 & 0.7861 \\
DMF & Microp & MiniCalW & 12.2 & 12.35 & -0.16 & $<0.0001$ & -0.57 & 0.5725 \\
DMF & Grav & MiniCalW & 12.1 & 12.35 & -0.26 & $<0.0001$ & -1.03 & 0.3048 \\
\hline
\end{tabular}

pHomVar: p-value variance homogeneity

$\mathrm{fd}$ : freedom degree reduction due to non homogeneity of variance was not indicated due to absence of significant difference

Only the comparisons between the calibrations obtained by Mic, MiniCal, MiniCalW and Grav were significant among them. The average of all the differences between the MFEC measurements and the different calibrations is $0.43 \mu \mathrm{m}$, but the difference between the device calibrations (Mic, Microp and Grav) that were not significant in the Student's T test of independent samples only reached at $0.20 \mu \mathrm{m}$. Although, on the other hand, the differences between the calibrations of devices (Mic, MiniCal and MiniCalW) that were significant $(p<0.05)$ yielded a higher value of $0.65 \mu \mathrm{m}$.

When the direct measurements (devices) are compared with the MFEC measurements calibrated by the same means of the direct measurements, significant results are only obtained in the case of the MiniCalW variable and in two Microp measurements. This will be presented in the Table 6 .

Table 6 Comparison of direct measurements with MFEC measurements with their respective calibration

\begin{tabular}{|c|c|c|c|c|c|c|c|}
\hline Sample & Description & Device & MFD (I) & SD (I) & MFD (2) & SD (2) & Sig \\
\hline I & $\mathrm{KI}$ & MiiniCal & 11.98 & 1.23 & 12.1 & 1.5 & ns \\
\hline 2 & KIb & MiiniCal & 11.98 & 1.23 & 12.14 & 1.5 & ns \\
\hline 3 & KO & MiiniCal & 11.98 & 1.23 & 11.55 & 1.36 & ns \\
\hline 4 & $\mathrm{KOb}$ & MiiniCal & 11.98 & 1.23 & 11.58 & 1.34 & ns \\
\hline 5 & $\mathrm{KI}$ & MiiniCalW & 12.35 & 0.23 & 11.72 & 1.36 & $* * *$ \\
\hline 6 & $\mathrm{klb}$ & MiiniCalW & 12.35 & 0.23 & 11.73 & 1.35 & $* * *$ \\
\hline 7 & $\mathrm{KO}$ & MiiniCalW & 12.35 & 0.23 & 11.72 & 1.37 & $* * *$ \\
\hline 8 & $\mathrm{kOb}$ & MiiniCalW & 12.35 & 0.23 & 11.66 & 1.32 & $* * *$ \\
\hline
\end{tabular}




\begin{tabular}{|c|c|c|c|c|c|c|c|}
\hline Sample & Description & Device & MFD (I) & SD (I) & MFD (2) & SD (2) & Sig \\
\hline 9 & $\mathrm{KI}$ & Microp & 12.38 & 1.82 & 11.89 & 1.42 & $*$ \\
\hline 10 & KIb & Microp & 12.38 & 1.82 & 11.89 & 1.42 & $*$ \\
\hline II & $\mathrm{k} 0 \mathrm{~b}$ & Microp & 12.38 & 1.82 & 12.25 & 1.38 & ns \\
\hline 12 & KO & Microp & 12.38 & 1.82 & 12.28 & 1.38 & ns \\
\hline 13 & KO & Microsc & 12.09 & 2.99 & 11.49 & 1.34 & ns \\
\hline 14 & KOb & Microsc & 12.09 & 2.99 & 11.53 & 1.37 & ns \\
\hline 15 & $\mathrm{KI}$ & Grav & 12.01 & 0.89 & 12.19 & 1.42 & ns \\
\hline 16 & KIb & Grav & 12.01 & 0.89 & 12.24 & 1.44 & ns \\
\hline 17 & KO & Grav & 12.01 & 0.89 & 11.91 & 1.37 & ns \\
\hline 18 & $\mathrm{KOb}$ & Grav & 12.01 & 0.89 & 11.92 & 1.39 & ns \\
\hline Total & & & 12.14 & 1.46 & 11.83 & 1.39 & ns \\
\hline
\end{tabular}

Reference:

MFD (I): direct measurement in the respective device

MFD (2): measurement by MFEC calibrated with the same mean of the respective device

SD (I): standard deviation of MFD (I)

SD (2): standard deviation of MFD (2)

Sig: significative diference of T Student independiente sampled:

Ns: non signficative $(>0.05)$

*: signficative $(p<0.05)$

$* * *$ : Signficiative $(p<0.001)$

When the average of the total differences between the direct measurement and the MFEC measurements is expressed, $0.32 \mu \mathrm{m}$ is obtained. Nevertheless, when only the comparisons that were significant are used $(\mathrm{p}<0.05: \mathrm{p}<0.001)$ an average difference of $0.59 \mu \mathrm{m}$ is obtained, and when the mean of the non-significant comparisons is estimated ( $p>0.05)$, a much lower value of $0.27 \mu \mathrm{m}$ is obtained.

\section{Results of the simulation of the data obtained by MiniFiber corrected for the errors of the direct measurements}

Results of the simulation of the data obtained by MiniFiber corrected for the errors of the direct measurements, by the results of the simulation with the inverse normal function are recorded using the standard error of the indirect measurement as an indicator of the systematic error of the measure of the Mini Fiber. The variable $\pm \mathrm{SE} *$ 1.96 is the confidence interval obtained with the residual values of the regression and is similar to the difference value obtained in Table 1. Then the result of the CI variable (95\%) is interesting, which reflects the amplitude of the IC, which if expressed on the mean obtained in the Mini fiber measurement results in EE/Average, which yields a value of $5 \% \mu \mathrm{m}$ tolerable error in any laboratory measurement. The correlation (expressed as a coefficient of determination) between the MFD measurement of the MFEC and the variable obtained by adding the error of the original measurement in the respective device, gave high results for the majority, but low for MiniCalW and Microp (first part). These figures were respectively R: 0.96-0.98 and 0.640.67 respectively. This would indicate that calibration based on direct measurements is more accurate as well as more accurate than indirect measurements.
It should be clarified that this error is established in terms of accuracy or systematic bias, that is, in relation to direct measurement.

\section{Discussion}

The bias that concerns animal fiber metrologists is one of precision and accuracy. In the case of precision, it has been shown that MFEC presents high precision and repeatability of the measurement, however, the accuracy lacked until now reliable measurements because they were comparisons with other devices (secondary measurements). In this work, primary measurements are introduced when measuring with a mini caliper, carrying out the gravimetric determination of the diameter, using a microscope with a higher resolution than the microprojector and face contrast, and finally the microprojector as a comparative.

\section{Data from direct measurements}

On the other hand, in the micron/pixel ratio, differences can be found where differences in mean diameter intervene and even in homogeneity of variance where the weighted average of the measurement with mini caliber intervenes, also indicating here that it is not a recommended variable for this measurement.

\section{Data of direct and indirect measurements and the comparison between them}

Comparison between direct measurements of Mini Fiber, calibrated over direct measurements The magnitude of the direct accuracy calculated by these methods is similar to that established in the form comparative with other devices and with animal fibers similar in diameter ${ }^{8}$ and similar to the reproducibility between Laserscan laboratories. ${ }^{5}$ 


\section{Results of the simulation of the data obtained by MiniFiber corrected for the errors of the direct measurements}

Although it does not present a normal distribution of the diameter of the Kevlar fiber, in any of the mid-sections, both the standard error and the confidence interval are also very similar to other measurements with the MiniFibre EC. ${ }^{13}$ However, the magnitude of the established error of the measurement should be taken with caution because it does not reflect the accuracy of the previous variable (EE/Med) but rather the precision of the measurement of the device, which however is very low because it is below the $1 \%$ in relation to the average.

\section{Calibration errors}

Calibration errors occur in all measurements except attribute counts. No calibration method is perfect and all calibrations have an associated uncertainty. Indeed, for some types of measurement, it is a requirement that calibration uncertainty be taken into account in estimates of measurement precision, although this notion does not appear to have been discussed in the wool standards. However, problems associated with calibration are discussed in the constituent parts of the international standard ISO 5725. In our type of measurement, the calibration error comprises two components: variability between different samples of the reference materials and variability due to the measurement processes used in calibration. The combined effect is a bias. Over a period of time, biases are generally canceled out and can be considered random errors, but in practice, if two measurements are carried out on different instruments, whether of the same type or not, each measurement will have a bias component, due to the calibration errors of each instrument.

In the case of airflow, OFDA and FDA or Laserscan, the calibration methods are currently all indirect and require checks to be performed on the instruments during or after calibration. However, the lack of a wide range of suitable calibration materials has limited the application of true verification procedures using materials other than those used for calibration. Therefore, current methods carry the danger that if the calibration samples are not representative, there could be significant bias from this source. Sometimes, while there were differences between samples, they were generally not enough to give a significant variation between instrument calibrations.

However, just because the calibration reference material samples are effectively the same does not mean that the instrument's calibrations will be the same. Significant differences have been observed in these data and, depending on the mean diameter, it is not uncommon to find biases of approximately 0.3 microns between laboratories. This point was reinforced by an investigation by the Australian Wool Surveillance Authority. ${ }^{28}$

In the case of the projection microscope, the calibration process is theoretically traceable to international metrology standards, but in practice, the IWTO- 8 is vague with regard to precision and traceability requirements, and so far it appears to have been paid little attention to this problem. Review of published statistics shows that differences of 0.5 microns or more between laboratories are not usual. ${ }^{12}$

In this case, the variability between different samples of the reference materials does not occur because it is the same sample of the same material; therefore, the variability will be due to the measurement processes used in the calibration and the instruments of origin of those measurements. ${ }^{10-19}$

\section{Conclusion}

It is possible to calibrate the MFEC instrument on a direct measurement of a non-deformable synthetic fiber that allows direct measurements of its thickness, and validate by comparisson between others instrument measure of different type of animal fibers. Measurement results with MiniCal and the Grav method can be made with an organic synthetic fiber such as the aramid Kevlar fibre and the accuracy of the MFEC measurement can be established in this way.

The bias of MF EC with respect to direct measurements with which the measurement difference is not significant is $0.28 \mu \mathrm{m}$, with which it is significant it is 0.59 and for all measurements taken together it is $0.32 \mu \mathrm{m}$. It can be assumed that this bias is not the random error of the device's measurement; therefore, it is a measure of its accuracy.

It is essential to calibrate the MFEC for each type of fiber to be measured, paying special attention to the preparation of the specimen to be measured.

\section{Acknowledgments}

The authors thank the company MaxCorp Ltd., Lima, Peru, for the provision of the MiniFiber EC equipment, its set-up and subsequent maintenance, without which this work would not have been possible.

\section{Funding}

None.

\section{Conflicts of interest}

The absence of any conflict of interest arisen between the company that owns the equipment used, the other equipment with which the results were compared, and the authors themselves is declared.

\section{References}

1. Qi K, Lupton C, Pfeiffer F, et al. Automatic image analysis system for objective measurement of animal fibers. Sheep Goat Res J. 1995;11(2):7177.

2. Li Q, Huang C, Bai L. Method and system for measuring wool fiber fineness measurement. Patent No CN1359006-A. China; 2002.

3. Baltuano O, Rojas J, Aching J, et al. Prototipo de fibrómetro digitial computarizado para medición automática del espesor de fibra de alpaca. Informe Científico Tecnológico. IPEN; 2005.

4. Brims M. Fibre assessment apparatus and method. Patent No W0 2007/025350 A1. Australia; 2006.

5. Sommerville P. Fundamental principles of fibre fineness measurement: Part 1: The Technical \& Commercial Requirements of Wool Testing Systems. Victoria, Australia: AWTA Ltd; 2001.

6. Cottle D, Baxter B. Wool metrology research and development to date Text Progress. 2015;47(3):163-315.

7. Quispe EC, Rubio MJ, Sacchero D, et al. Interlaboratory test performance of a portable fiber tester, tekstil ve mühendis. 2019;26:330-334.

8. Quispe MD, Benavidez G, Sauri RA, et al. Development and preliminary validation of an automatic digital analysis system for animal fibre analysis. South African J Anim Scie. 2017;47(6):822-833.

9. Hazelton N. Instrument calibration for the 21st century. 57th Annual Meeting of Minnesota Society of Professional Surveyors (MSPS). St. Cloud, Minnesota; 2009. 
10. Scobie DR, Merrick NC, Saville DJ. Comparison of methods for measuring fibre diameter of Rommer wool. Proc New Zeal Soc Anim Prod. 1994;54:155-157.

11. Baxter BP. Comparison of Laserscan, OFDA and airflow on raw wool samples. IWTO Raw Wool Group Report RWG02. Nice, France; 1998.

12. Baxter P, Marler J. The 2003 Australian wool innovation on-farm fibre measurement instrument evaluation trial. Part 2: Performance in objective classing and ranking for animal selection. Wool Tech Sheep Breed. 2004;52(2):124-170.

13. Quispe EC, Quispe MD. Minifiber Y Fiber Den: Innovaciones tecnológicas para la evaluación de fibras y pieles de animales. Libro de Resúmenes del I Congreso Internacional de Investigacipones Tecnológica Agropecuarias. Chota, Perú; 2018.

14. Walker JW, Stewart WC, Pope R, et al. Evaluation of mean fiber diameter measurements by FibreLux micron meter and OFDA 2000 in Texas and Intermountain West wool. Small Rumin Res. 2018;159:31-37.

15. Sommerville PJ. Fundamental principles of fibre fineness measurement: Part 3: Technologies for measuring fineness of wool fibre. AWTA Ltd, Victoria, Australia; 2002.

16. Lunney HW, Brown GH. Reference standard wool tops for the measuremente of fiber diameter distribution. Text Res J. 1985;55(11):671676.

17. Sommerville P. Fundamental principles of fibre fineness measurement: Part 6: Direct Measurement. AWTA Ltd, Victoria, Australia; 2003.

18. Kawabata S. Measurement of the transverse mechanical properties of high-performance fibers. J Text Inst. 1990;81;432-439.

19. Marler JW, Hansford KA, McLachlan IM. The precision of OFDA 2000 and FLEECESCAN for estimating the diameter characteristics of fleeces: A case study. Wool Tech Sheep Breed. 2002;50(4):832-836.
20. Marler JW, Baxter BP. The 2003 Australian wool innovation on-farm fibre measurement instrument evaluation trial. Part 1: Accuracy and precision trials. Evian Meeting of the International Wool Textile Organisation (IWTO). Report No CTF 01, France; 2004.

21. Baxter P, Marler J. The 2003 Australian wool innovation on-farm fibre measurement instrument evaluation trial. Part 2: Performance in objective classing and ranking for animal selection. Wool Tech Sheep Breed. 2004;52(2):124-170.

22. Heath W, Barkhuizen J, Wright O. The relationship between mean fibre diameter measurements by Airflow and Laserscan for South African wools. In: Cairo Meeting of the International Wool Textile Organization Wellington (IWTO), Egypt. 2006.

23. JCGM 200:2008 International vocabulary of metrology - Basic and general concepts and associated terms (VIM) JCGM 200:2008 International vocabulary of metrology.

24. Taylor JR. An introduction to error analysis: the study of uncertainties in physical measurements. University Science Books. 1999:128-129.

25. Andrews MW, David HG. Fibre-diameter measurement by projection microscope: precision of the mean. J Text Inst. 1978;69(12):391-394.

26. Burns RH. The micrometer caliper for measuring wool fibres. Wyoming Agric Expt Sta. 1935;204:36.

27. von Bergen, W. Measurement of fibre widths by the wedge method. Melliand Textile Monthly, 1932.

28. AWSA. Investigations of reported fibre diameter differences between two laboratories, Public release statement, 1990. 